PROCEEDINGS OF THE

AMERICAN MATHEMATICAL SOCIETY

Volume 134, Number 1, Pages 253-258

S 0002-9939(05)08031-7

Article electronically published on June 14, 2005

\title{
REPRESENTING CONDITIONAL EXPECTATIONS AS ELEMENTARY OPERATORS
}

\author{
RAJESH PEREIRA
}

(Communicated by David R. Larson)

\begin{abstract}
Let $\mathcal{A}$ be a $C^{*}$-algebra and let $\mathcal{B}$ be a $C^{*}$-subalgebra of $\mathcal{A}$. We call a linear operator from $\mathcal{A}$ to $\mathcal{B}$ an elementary conditional expectation if it is simultaneously an elementary operator and a conditional expectation of $\mathcal{A}$ onto $\mathcal{B}$. We give necessary and sufficient conditions for the existence of a faithful elementary conditional expectation of a prime unital $C^{*}$-algebra onto a subalgebra containing the identity element. We give a description of all faithful elementary conditional expectations. We then use these results to give necessary and sufficient conditions for certain conditional expectations to be index-finite (in the sense of Watatani) and we derive an inequality for the index.
\end{abstract}

\section{ELEMENTARY OPERATORS}

Let $\mathcal{A}$ be a $C^{*}$-algebra. $\Phi: \mathcal{A} \rightarrow \mathcal{A}$ is called an elementary operator if either $\Phi=0$ or there exists $\left\{x_{i}\right\}_{i=1}^{n} ;\left\{y_{i}\right\}_{i=1}^{n}$, two finite sets each containing the same number of linearly independent elements in $\mathcal{A}$, such that $\Phi(a)=\sum_{i=1}^{n} x_{i} a y_{i}$. A $C^{*}$-algebra $\mathcal{A}$ is called prime if for any non-zero $x, y \in \mathcal{A}$, there exists $z \in \mathcal{A}$ such that $x z y \neq 0$. It was first noted by Mathieu 4 that elementary operators on prime $C^{*}$-algebras have many interesting properties. Many of the results in section 4 of [4] will be used later in this paper. We will also need the following results.

Lemma 1.1. Let $\mathcal{A}$ be a prime $C^{*}$-algebra, let $\left\{x_{i}\right\}_{i=1}^{n}$ and $\left\{y_{i}\right\}_{i=1}^{n}$ be two linearly independent sets in $\mathcal{A}$ and let $c_{i j} \in \mathbb{C}$ for $1 \leq i, j \leq n$. Then $\sum_{1 \leq i, j \leq n} c_{i j} x_{i} m y_{j}=0$ for all $m \in \mathcal{A}$ implies that $c_{i j}=0$ for all $i, j$.

Proof. Let $C$ be the $n$ by $n$ matrix whose $(i, j)$ entry is $c_{i j}$. Let $C=U D V$ be its singular value decomposition, where $U$ and $V$ are unitary matrices and $D$ is a diagonal matrix with non-negative entries. Now let $a_{k}=\sum_{i=1}^{n} u_{i k} x_{i}$ and $b_{k}=\sum_{j=1}^{n} v_{k j} y_{j}$. Note that $\left\{a_{k}\right\}_{k=1}^{n}$ and $\left\{b_{k}\right\}_{k=1}^{n}$ are both linearly independent sets and $\sum_{k=1}^{n} d_{k k} a_{k} m b_{k}=0$ for all $m \in \mathcal{A}$. Hence Theorem 4.1 of [4] implies that $C=0$.

Any completely positive elementary operator $\Phi$ has a representation of the form $\Phi(a)=\sum_{i=1}^{n} x_{i}^{*} a x_{i}$ for some linearly independent set of elements $\left\{x_{i}\right\}_{i=1}^{n}$ ([4],

Received by the editors September 1, 2004.

2000 Mathematics Subject Classification. Primary 46L05, 47B47.

Key words and phrases. Prime $C^{*}$-algebras, conditional expectations, elementary operators, index finite type, minimal conditional expectation.

(C)2005 American Mathematical Society Reverts to public domain 28 years from publication 
Theorem 4.10). Since any conditional expectation is completely positive, we will only consider elementary operators of this form from here on.

Corollary 1.2. Let $\mathcal{A}$ be a prime $C^{*}$-algebra. Let $V$ be a finite-dimensional subspace of $\mathcal{A}$ endowed with an inner product $\langle\cdot, \cdot\rangle$. Let $\left\{x_{i}\right\}_{i=1}^{n}$ be an orthonormal basis of $V$ and let $\left\{y_{k}\right\}_{k=1}^{n}$ be any $n$-tuple of elements in $V$. Then $\sum_{i=1}^{n} x_{i}^{*} a x_{i}=$ $\sum_{k=1}^{n} y_{k}^{*} a y_{k}$ for all $a \in A$ if and only if $\left\{y_{k}\right\}_{k=1}^{n}$ is also an orthonormal basis of $V$.

Proof. Suppose $\sum_{i=1}^{n} x_{i}^{*} a x_{i}=\sum_{k=1}^{n} y_{k}^{*} a y_{k}$. We can write $y_{k}=\sum_{i=1}^{n} c_{i k} x_{i}$ where $c_{i k}=\left\langle y_{k}, x_{i}\right\rangle$. Let $C$ denote the $n$ by $n$ matrix with the $(i, j)^{\text {th }}$ entry equal to $c_{i j}$ for all $i, j$. Then $\sum_{i=1}^{n} x_{i}^{*} a x_{i}=\sum_{k=1}^{n} y_{k}^{*} a y_{k}=\sum_{i, j=1}^{n}\left[C C^{*}\right]_{i j} x_{j}^{*} a x_{i}\left(\left[C C^{*}\right]_{i j}\right.$ is the $(i, j)^{\text {th }}$ entry of the matrix $\left.C C^{*}\right)$ and hence $\sum_{i, j=1}^{n}\left[C C^{*}-I\right]_{i j} x_{j}^{*} a x_{i}=0$. $C$ is therefore unitary by the previous lemma and $\left\{y_{k}\right\}_{k=1}^{n}$ is an orthonormal basis. The converse may be proved by reversing our steps.

\section{Conditional expectations}

We begin by recalling the concept of a conditional expectation onto a subalgebra first introduced in 5 .

Definition 2.1. Let $\mathcal{A}$ be a $C^{*}$-algebra and let $\mathcal{B} \subseteq \mathcal{A}$ be a $C^{*}$-subalgebra. Then we call $E: \mathcal{A} \rightarrow \mathcal{B}$ a conditional expectation of $\mathcal{A}$ onto $\mathcal{B}$ if it satisfies the following three properties:

(1) $E(b)=b, \forall b \in \mathcal{B}$,

(2) $E\left(b_{1} a b_{2}\right)=b_{1} E(a) b_{2} \quad \forall a \in \mathcal{A}, \forall b_{1}, b_{2} \in \mathcal{B}$,

(3) $a \geq 0 \Longrightarrow E(a) \geq 0$ for all $a \in \mathcal{A}$.

We say that $E$ is a faithful conditional expectation if, in addition to the three conditions above, $E$ satsifies the following:

(4) If $E\left(a^{*} a\right) \neq 0$ for all non-zero $a \in \mathcal{A}$.

We say that $E$ is an elementary conditional expectation of $\mathcal{A}$ onto $\mathcal{B}$, if $E$ is an elementary operator on $\mathcal{A}$ which is also a conditional expectation of $\mathcal{A}$ onto $\mathcal{B}$.

Conditional expectations may not be unique. If $\mathcal{A}$ has a faithful tracial state $\tau$, there is a unique conditional expectation of $\mathcal{A}$ onto $\mathcal{B}$ satisfying $\tau(E(a))=\tau(a)$ for all $a \in \mathcal{A}$ which is called the $\tau$-preserving conditional expectation. If we consider $\mathcal{A}$ to be a Hilbert space with the inner product $\langle x, y\rangle=\tau\left(y^{*} x\right)$, the $\tau$-preserving conditional expectation is the orthogonal projection on the subspace $\mathcal{B}$.

\section{Finite-Dimensional $C^{*}$-Algebras}

Let $\mathcal{M}$ be a finite-dimensional $C^{*}$-algebra (the identity element will be denoted $1_{\mathcal{M}}$ or just 1 if it is clear which $C^{*}$-algebra it belongs to). It is well known that every finite-dimensional $C^{*}$-algebra is isomorphic to a direct sum of matrix algebras; hence $\mathcal{M} \cong \bigoplus_{k=1}^{q} M_{n_{k}}(\mathbb{C})$ for some finite set of natural numbers $\left\{n_{k}\right\}_{k=1}^{q}$. We can form a basis of $\mathcal{A}$ consisting of matrix units $\left\{\left\{e_{i j}^{(k)}\right\}_{i, j=1}^{n_{k}}\right\}_{k=1}^{q}$ satisfying $e_{i j}^{(k)} e_{r s}^{(t)}=$ $\delta_{j r} \delta_{k t} e_{i s}^{(t)}$ and $e_{i j}^{(k) *}=e_{j i}^{(k)}$. We note that there are $q$ minimal central projections of $\mathcal{M}$, and they are of the form $c_{k}=\sum_{i=1}^{n_{k}} e_{i i}^{(k)}$. The span of the minimal central projections is $Z(\mathcal{M})$, the center of $\mathcal{M}$. We note that the minimal central projections are independent of the choice of matrix units. We will define the left-regular trace $\tau$ on $\mathcal{M}$ as follows: $\tau\left(c_{k}\right)=n_{k}^{2}$ (hence $\tau(1)=\operatorname{dim}(\mathcal{M})$ and $\tau\left(e_{i j}^{(k)}\right)=\delta_{i j} n_{k}$ for any set of matrix units). The terminology here comes from the fact that this $\tau$ can be 
viewed as the trace (sum of the diagonal matrix entries) of the left regular matrix representation of $\mathcal{M}$ (see pg. 424 of [1] for more details), although we will not use this fact in what follows. We end this section with the following useful elementary result.

Lemma 3.1. Let $\mathcal{M}$ be a finite-dimensional $C^{*}$-algebra and let $I$ be a proper right ideal of $\mathcal{M}$. Then there exists a non-zero $m \in \mathcal{M}$ such that $m I=0$.

\section{Faithiful elementary conditional expectations}

We recall that if $\mathcal{B}$ is a $C^{*}$-subalgebra of a $C^{*}$-algebra $\mathcal{A}, B^{\prime} \cap \mathcal{A}=\{a \in \mathcal{A} \mid a b=$ $b a, \forall b \in \mathcal{B}\}$ and is called the relative commutant of $\mathcal{B}$ in $\mathcal{A}$.

Lemma 4.1. Let $\mathcal{A}$ be a unital prime $C^{*}$-algebra and let $\mathcal{B}$ be a $C^{*}$-subalgebra of $\mathcal{A}$ with $1_{\mathcal{A}} \in \mathcal{B}$. Suppose there exists $E$, a faithful elementary conditional expectation from $\mathcal{A}$ to $\mathcal{B}$ and let $\left\{x_{i}\right\}_{i=1}^{n}$ be a linearly independent set of elements in $\mathcal{A}$ such that $E(a)=\sum_{i=1}^{n} x_{i}^{*} a x_{i}$. Then $\mathcal{B}^{\prime} \cap \mathcal{A}=\operatorname{span}\left(\left\{x_{i}\right\}_{i=1}^{n}\right)$. (In particular $\mathcal{B}^{\prime} \cap \mathcal{A}$ must be finite dimensional).

Proof. First note that $E(a b)=E(a) b$ for all $a \in \mathcal{A}$ and all $b \in \mathcal{B}$ and hence $\sum_{i=1}^{n} x_{i}^{*} a\left(x_{i} b-b x_{i}\right)=0$. Therefore, by Theorem 4.1 of [4], $x_{i} \in \mathcal{B}^{\prime} \cap \mathcal{A}$ for all $i$. Now let $u$ be a unitary element in $\mathcal{B}^{\prime} \cap \mathcal{A}$. Then $u E(a)=E(a) u$ for all $x \in \mathcal{A}$. Hence $\sum_{i=1}^{n} u x_{i}^{*} a x_{i}=\sum_{i=1}^{n} x_{i}^{*} a x_{i} u$ and by Corollary 4.7 of [4], $\operatorname{span}\left(\left\{x_{i} u\right\}_{i=1}^{n}\right)=$ $\operatorname{span}\left(\left\{x_{i}\right\}_{i=1}^{n}\right)$. Since every element in a unital $C^{*}$-algebra can be expressed as a linear combination of four unitaries, $\operatorname{span}\left(\left\{x_{i}\right\}_{i=1}^{n}\right)$ is a right ideal in $\mathcal{B}^{\prime} \cap \mathcal{A}$. Similarly, $\operatorname{span}\left(\left\{x_{i}^{*}\right\}_{i=1}^{n}\right)$ is a left ideal and hence $\operatorname{span}\left(\left\{x_{i}^{*} x_{j}\right\}_{i, j=1}^{n}\right)$ is a two-sided ideal which contains the identity and hence is equal to $\mathcal{B}^{\prime} \cap \mathcal{A}$. In particular, $\mathcal{B}^{\prime} \cap \mathcal{A}$ is a finite-dimensional $C^{*}$-algebra. Hence, if $\operatorname{span}\left(\left\{x_{i}\right\}_{i=1}^{n}\right)$ is a proper right ideal of $\mathcal{B}^{\prime} \cap \mathcal{A}$, then there exists a non-zero $m \in \mathcal{B}^{\prime} \cap \mathcal{A}$ such that $m x_{i}=0$ for all $i$. But then $E_{\mathcal{B}}\left(m^{*} m\right)=0$ which contradicts faithfulness. So $\mathcal{B}^{\prime} \cap \mathcal{A}=\operatorname{span}\left(\left\{x_{i}\right\}_{i=1}^{n}\right)$.

Theorem 4.2. Let $\mathcal{A}$ be a unital prime $C^{*}$-algebra and let $\mathcal{B}$ be a $C^{*}$-subalgebra of $\mathcal{A}$ with $1_{\mathcal{A}} \in \mathcal{B}$. Then there exists $E$, a faithful elementary conditional expectation of $\mathcal{A}$ onto $\mathcal{B}$ if and only if $\mathcal{B}$ is the relative commutant of a finite-dimensional $C^{*}$-subalgebra of $\mathcal{A}$ containing $1_{\mathcal{A}}$.

Proof. Let $E(a)=\sum_{i=1}^{n} x_{i}^{*} a x_{i}$ be a conditional expectation of $\mathcal{A}$ onto $\mathcal{B}$. Let $\mathcal{M}=\mathcal{B}^{\prime} \cap \mathcal{A} ; \mathcal{M}$ is finite dimensional by the previous lemma and $\mathcal{B} \subseteq \mathcal{M}^{\prime} \cap \mathcal{A}$. Then $\sum_{i=1}^{n} x_{i}^{*} x_{i}=1$ and $E(a)=a$ for all $a \in \mathcal{M}^{\prime} \cap \mathcal{A}$. Hence $\mathcal{B}=\mathcal{M}^{\prime} \cap \mathcal{A}$.

Now suppose $\mathcal{B}=\mathcal{M}^{\prime} \cap \mathcal{A}$, where $\mathcal{M} \cong \bigoplus_{k=1}^{q} M_{n_{k}}(\mathbb{C})$. Now consider the elementary operator $E_{0}(a)=\sum_{k=1}^{q} \frac{1}{n_{k}} \sum_{i, j=1}^{n_{k}} e_{j i}^{(k)} a e_{i j}^{(k)}$, where $\left\{\left\{e_{i j}^{(k)}\right\}_{i, j=1}^{n_{k}}\right\}_{k=1}^{q}$ are a set of matrix units for $\mathcal{M}$. Note that $e_{r s}^{(t)} E_{0}(a)=\frac{1}{n_{t}} \sum_{i, j=1}^{n_{t}} e_{r i}^{(t)} a e_{i s}^{(t)}=E_{0}(a) e_{r s}^{(t)}$ so $E_{0}(a) \in \mathcal{B}$. We also have $E_{0}(1)=\sum_{k=1}^{q} \sum_{j=1}^{n_{k}} e_{j j}^{(k)}=1$. Now it can easily be verified that $E_{0}$ satisfies all of the conditions of a faithful conditional expectation. If $\mathcal{A}$ has any tracial states, it is easy to see that $E_{0}$ is the unique trace-preserving conditional expectation for any trace on $\mathcal{A}$.

We note that the "if" direction of the above result remains true even if $\mathcal{A}$ is not prime. We can show that $E_{0}$ is independent of the choice of matrix units by noting that any set of normalized matrix units $\left\{\left\{n_{k}^{-\frac{1}{2}} e_{i j}^{(k)}\right\}_{i, j=1}^{n_{k}}\right\}_{k=1}^{q}$ is an orthonormal basis of $\mathcal{M}$ with respect to the inner product $\langle x, y\rangle=\tau\left(y^{*} x\right)$, where $\tau$ is the 
left regular trace of $\mathcal{M}$ and then by using Corollary 1.2. We will call $E_{0}(a)=$ $\sum_{k=1}^{q} \frac{1}{n_{k}} \sum_{i, j=1}^{n_{k}} e_{j i}^{(k)} a e_{i j}^{(k)}$ the minimal conditional expectation of $\mathcal{A}$ onto $\mathcal{B}$ (in the next section, we will explain the reason for calling $E_{0}$ minimal). We can now characterize all faithful elementary conditional expectations in terms of $E_{0}$.

Theorem 4.3. Let $\mathcal{A}$ be a unital prime $C^{*}$-algebra, let $\mathcal{M}$ be a finite-dimensional $C^{*}$-subalgebra of $\mathcal{A}$ with $1_{\mathcal{A}} \in \mathcal{M}, \mathcal{B}=\mathcal{M}^{\prime} \cap \mathcal{A}$ and let $E_{0}$ be the minimal conditional expectation of $\mathcal{A}$ onto $\mathcal{B}$. Let $E(x)=\sum_{i=1}^{n} x_{i}^{*} a x_{i}$ where the $\left\{x_{i}\right\}_{i=1}^{n}$ is a basis of $\mathcal{M}$. Then $E$ is a faithful conditional expectation of $\mathcal{A}$ onto $\mathcal{B}$ if and only if $E(x)=E_{0}(r x r)$ for some positive invertible $r \in \mathcal{M}$ such that $E_{0}\left(r^{2}\right)=1$.

Proof. We note that the "if" direction can easily be seen to be true; we will only prove the "only if" direction. Suppose $E$ is a faithful conditional expectation of $\mathcal{A}$ onto $\mathcal{B}$ and let $\langle\cdot, \cdot\rangle$ be the inner product on $\mathcal{M}$ defined by $\left\langle x_{i}, x_{j}\right\rangle=\delta_{i j}$. We note that $x_{i} m=\sum_{j=1}^{n}\left\langle x_{i} m, x_{j}\right\rangle x_{j}$ and $m x_{j}^{*}=\left(x_{j} m^{*}\right)^{*}=\sum_{i=1}^{n}\left\langle x_{i}, x_{j} m^{*}\right\rangle x_{i}^{*}$. Since $E$ maps $\mathcal{A}$ onto $\mathcal{B}, \sum_{i, j=1}^{n}\left\langle x_{i}, x_{j} m^{*}\right\rangle x_{i}^{*} a x_{j}=\sum_{j=1}^{n} m x_{j}^{*} a x_{j}=\sum_{i=1}^{n} x_{i}^{*} a x_{i} m=$ $\sum_{i, j=1}^{n}\left\langle x_{i} m, x_{j}\right\rangle x_{i}^{*} a x_{j}$ for all $m \in \mathcal{M}$. Hence the inner product has the property that $\langle x m, y\rangle=\left\langle x, y m^{*}\right\rangle$ for all $x, y, m \in \mathcal{M}$ and there exists an positive invertible $r \in \mathcal{M}$ such that $\langle x, y\rangle=\tau\left(y^{*} r^{-2} x\right)$, where $\tau$ is the left regular trace on $\mathcal{M}$. Therefore the set $\left\{\left\{\frac{1}{n_{k}} r e_{i j}^{(k)}\right\}_{i, j=1}^{n_{k}}\right\}_{k=1}^{q}$ is an orthonormal basis with respect to our inner product and $E(x)=E_{0}(r x r)$ by Corollary 1.2 and $E_{0}\left(r^{2}\right)=E(1)=1$.

\section{INDEX FINITENESS}

Definition 5.1. Let $\mathcal{A}$ be a $C^{*}$-algebra, let $\mathcal{B}$ be a $C^{*}$-subalgebra of $\mathcal{A}$ and let $E$ be a conditional expectation of $\mathcal{A}$ onto $\mathcal{B}$. A finite set of pairs of elements in $\mathcal{A}$, $\left\{\left(u_{i}, v_{i}\right)\right\}_{i=1}^{n}$ is called a quasi-basis for $E$ if $\sum_{i=1}^{n} u_{i} E\left(v_{i} a\right)=a=\sum_{i=1}^{n} E\left(a u_{i}\right) v_{i}$ for any $a \in \mathcal{A}$. A conditional expectation $E$ is said to be of index-finite type if there exists a quasi-basis for $E$.

We note that the quasi-basis may not be unique. An index-finite $E$ always admits a quasi-basis of the form $\left\{\left(u_{i}, u_{i}^{*}\right)\right\}_{i=1}^{n}$ ([్], Lemma 2.1.6). In the special case where $\mathcal{B}^{\prime} \cap \mathcal{A}$ is finite dimensional, if one conditional expectation of $\mathcal{A}$ onto $\mathcal{B}$ is of index-finite type, then every faithful conditional expectation of $\mathcal{A}$ onto $\mathcal{B}$ is of index-finite type ([6], Proposition 2.10.2). In the following theorem, $\mathbb{C}$ is the $C^{*}$-subalgebra of $\mathcal{A}$ consisting of all scalar multiples of the identity element.

Theorem 5.2. Let $\mathcal{A}$ be a unital prime $C^{*}$-algebra, let $\mathcal{M}$ be a finite-dimensional $C^{*}$-subalgebra of $\mathcal{A}$ with $1_{\mathcal{A}} \in \mathcal{M}$, and let $\mathcal{B}=\mathcal{M}^{\prime} \cap \mathcal{A}$. Further, let $\tau$ denote the left regular trace of $\mathcal{M}$. Then the following are equivalent:

(1) Any faithful conditional expectation of $\mathcal{A}$ onto $\mathcal{B}$ is of index-finite type.

(2) There exists $F$, an elementary operator on $\mathcal{A}$, such that $\left.F\right|_{\mathcal{M}}$ is the $\tau$ preserving faithful conditional expectation of $\mathcal{M}$ onto $\mathbb{C}$.

(3) There exists $G$, an elementary operator on $\mathcal{A}$, such that $\left.G\right|_{Z(\mathcal{B})}$ is the $\tau$ preserving faithful conditional expectation of $Z(\mathcal{B})$ onto $\mathbb{C}$.

(4) No proper two-sided ideal of $\mathcal{A}$ contains a minimal central projection of $\mathcal{B}$.

If any of these four equivalent conditions hold, then any conditional expectation of $\mathcal{A}$ onto $\mathcal{B}$ is an elementary conditional expectation.

Proof. (1) $\Longrightarrow(2)$ Let $\left\{x_{j}\right\}_{j=1}^{m}$ be an orthonormal basis of $\mathcal{M}$ with respect to the inner product $\langle x, y\rangle=\tau\left(y^{*} x\right)$ with $x_{1}=(\tau(1))^{-\frac{1}{2}} 1$. Now $E_{0}(a)=\sum_{j=1}^{m} x_{j}^{*} a x_{j}$ is 
the minimal conditional expectation of $\mathcal{A}$ onto $\mathcal{B}$. Let $\left\{\left(u_{i}, v_{i}\right)\right\}_{i=1}^{n}$ be a quasi-basis for $E_{0}$ and let $F(a)=\frac{1}{\tau(1)} \sum_{i=1}^{n} u_{i} a v_{i}$. Then

$$
a=\sum_{i=1}^{n} u_{i} E\left(v_{i} a\right)=\sum_{j=1}^{m} \sum_{i=1}^{n} \tau(1) F\left(x_{j}\right) a x_{j} .
$$

So $F\left(x_{i}\right)=0$ for $i=2,3, \ldots, n$ and $F(1)=1$. Hence $\left.F\right|_{\mathcal{M}}$ is the $\tau$-preserving faithful conditional expectation of $\mathcal{M}$ onto $\mathbb{C}$.

$(2) \Longrightarrow(1)$ Let $F(x)=\sum_{i=1}^{n} u_{i} a v_{i}$ where $\left\{u_{i}\right\}_{i=1}^{n}$ and $\left\{v_{i}\right\}_{i=1}^{n}$ are both linearly independent sets. We can show that $\left\{\left(u_{i}, v_{i}\right)\right\}_{i=1}^{n}$ is a quasi-basis for $E_{0}$ by reversing the steps of our previous argument.

$(2) \Longrightarrow$ (3) Simply take $G=F$.

(3) $\Longrightarrow(2)$ Let $E_{0}$ be the minimal conditional expectation of $\mathcal{A}$ onto $\mathcal{B}$. Note that $\left.E_{0}\right|_{\mathcal{M}}$ is a $\tau$-preserving conditional expectation of $\mathcal{M}$ onto $Z(\mathcal{B})$. Hence let $F(a)=G\left(E_{0}(a)\right)$ for all $a \in \mathcal{A}$.

$(3) \Longrightarrow$ (4) $G$ maps any minimal central projection of $\mathcal{B}$ into a multiple of the identity. Since any elementary operator will map a two-sided ideal into itself, no proper two-sided ideal of $\mathcal{A}$ contains a minimal central projection of $\mathcal{B}$.

(4) $\Longrightarrow$ (3) If any ideal of $\mathcal{A}$ contains a non-zero element of $Z(\mathcal{B})$, then it also contains a minimal central projection of $\mathcal{B}$. So any non-zero linear combination of the minimal central projections cannot be in any proper two-sided ideal of $\mathcal{A}$. The existence of $G$ now follows from Proposition 1.1 of $[3$.

The last sentence in the statement of the theorem is an immediate consequence of Proposition 2.10 .9 of 6 .

We note that the fourth of the equivalent conditions is satisfied in certain important cases such as when $\mathcal{A}$ is simple or when $Z(\mathcal{B})=\mathbb{C}$.

Watatani has defined the index of a conditional expectation $E$ having a quasibasis $\left\{\left(u_{i}, v_{i}\right)\right\}_{i=1}^{n}$ as follows: $\operatorname{ind}(E)=\sum_{i=1}^{n} u_{i} v_{i}$ and has shown that $\operatorname{ind}(E)$ is independent of the choice of quasi-basis and is in $Z(\mathcal{A})$ [6] (in our case, $\mathcal{A}$ is prime; so $Z(\mathcal{A})=\mathbb{C})$.

Theorem 5.3. Let $\mathcal{A}$ be a unital prime $C^{*}$-algebra, let $\mathcal{M}$ be a finite-dimensional $C^{*}$-subalgebra of $\mathcal{A}$ with $1_{\mathcal{A}} \in \mathcal{M}$, and let $\mathcal{B}=\mathcal{M}^{\prime} \cap \mathcal{A}$. Let $E$ be a faithful conditional expectation of $\mathcal{A}$ onto $\mathcal{B}$. If $E$ is index-finite, then $\operatorname{dim}(\mathcal{M}) \leq \operatorname{ind}(E)$ with equality if and only if $E$ is the minimal conditional expectation.

Proof. Let $E_{0}$ be the minimal conditional expectation of $\mathcal{A}$ onto $\mathcal{B}$ and let $\left\{\left(u_{i}, v_{i}\right)\right\}_{i=1}^{n}$ be a quasi-basis for $E_{0}$. By Theorem 4.3, $E(a)=E_{0}($ rar $)$ for some positive invertible $r \in \mathcal{M}$ such that $E_{0}\left(r^{2}\right)=1$. Then $\left\{\left(u_{i} r^{-1}, r^{-1} v_{i}\right)\right\}_{i=1}^{n}$ is a quasi-basis for $E$. Using an argument in the first paragraph of the proof of Theorem 5.2 $\operatorname{ind}(E)=\sum_{i=1}^{n} u_{i} r^{-2} v_{i}=\tau\left(r^{-2}\right)$, where $\tau$ is the left regular trace on $\mathcal{M}$. Now $\tau(1) \operatorname{dim}(\mathcal{M})=(\tau(1))^{2} \leq \tau\left(r^{2}\right) \tau\left(r^{-2}\right)=\tau\left(E_{0}\left(r^{2}\right)\right) \tau\left(r^{-2}\right)=\tau(1) \tau\left(r^{-2}\right)=$ $\tau(1) \operatorname{ind}(E)$. The inequality step follows from the Cauchy-Schwarz inequality, and hence equality holds only when $r=1$ and $E=E_{0}$.

$E_{0}$ minimizes the index and is therefore a minimal conditional expectation in the usual sense (as in section 2.12 of [6]), thus justifying our use of the terminology. If $\mathcal{A}$ has a tracial state, the conditional expectation which minimizes the index is also the one which preserves all the tracial states of $\mathcal{A}$. We also note that our inequality seems to be a Watatani index analogue of an inequality for the Jones index described in Jones' original paper (in the Remark after Corollary 2.2.3 in [2]). 
RAJESH PEREIRA

\section{REFERENCES}

[1] N. Jacobson, Basic Algebra I, 2nd Ed. W. H. Freeman and Company, New York, 1985. MR0780184 (86d:00001)

[2] V. F. R. Jones, Index for subfactors, Invent. Math., 72 (1983), 1-25. MR0696688 (84d:46097)

[3] B. Magajna, A transitivity theorem for algebras of elementary operators, Proc. Amer. Math. Soc., 118 (1993), 119-127. MR.1158004 (93f:46092)

[4] M. Mathieu, Elementary operators on prime $C^{*}$-algebras I, Math. Ann., 284 (1989), 223-244. MR:1000108 (90h:46092)

[5] H. Umegaki, Conditional expectation in an operator algebra, Tohoku. Math. J., 6 (1954), 177-181. MR0068751(16:936b)

[6] Y. Watatani, Index for $C^{*}$-subalgebras, Mem. Amer. Math. Soc., 83 (1990), no. 424. MR0996807 (90i:46104)

Department of Mathematics and Statistics, University of Saskatchewan, 106 Wiggins Road, Saskatoon, Saskatchewan, Canada S7N 5E6

E-mail address: rjxpereira@yahoo.com 